\title{
Skyrmion Ratchet in Funnel Geometries
}

\author{
J. C. Bellizotti Souza ${ }^{1}$, N. P. Vizarim ${ }^{2}$, C. J. O. Reichhardt ${ }^{3}$, C. Reichhardt ${ }^{3}$ and P. A. Venegas ${ }^{1}$ \\ 1 Departamento de Física, Faculdade de Ciências, \\ Unesp-Universidade Estadual Paulista, CP 473, 17033-360 Bauru, SP, Brazil \\ 2 POSMAT - Programa de Pós-Graduação em Ciência e Tecnologia de Materiais, \\ Faculdade de Ciências, Universidade Estadual Paulista - UNESP, Bauru, SP, CP 473, 17033-360, Brazil \\ 3 Theoretical Division and Center for Nonlinear Studies, \\ Los Alamos National Laboratory, Los Alamos, New Mexico 87545, USA
}

(Dated: May 25, 2021)

\begin{abstract}
Using a particle-based model, we simulate the behavior of a skyrmion under the influence of asymmetric funnel geometries and ac driving at zero temperature. We specifically investigate possibilities for controlling the skyrmion motion by harnessing a ratchet effect. Our results show that as the amplitude of a unidirectional ac drive is increased, the skyrmion can be set into motion along either the easy or hard direction of the funnel depending on the ac driving direction. When the ac drive is parallel to the funnel axis, the skyrmion flows in the easy direction and its average velocity is quantized. In contrast, when the ac drive is perpendicular to the funnel axis, a Magnus-induced ratchet effect occurs, and the skyrmion moves along the hard direction with a constant average velocity. For biharmonic ac driving of equal amplitude along both the parallel and perpendicular directions, we observe a reentrant pinning phase where the skyrmion ratchet vanishes. For asymmetric biharmonic ac drives, the skyrmion exhibits a combination of effects and can move in either the easy or hard direction depending on the configuration of the ac drives. These results indicate that it is possible to achieve controlled skyrmion motion using funnel geometries, and we discuss ways in which this could be harnessed to perform data transfer operations.
\end{abstract}

\section{INTRODUCTION}

The flow of particles interacting with asymmetric landscapes can be controlled by means of a ratchet effect, which is a net dc transport that emerges from a combination of broken spatial symmetry and an oscillating drive. The ratchet effect can also be understood in terms of a diode effect, where the asymmetry produces different depinning forces in different directions, yielding a preferential or "easy" direction of motion ${ }^{1}$. This effect has been investigated in several systems such as protein motors ${ }^{2} 4$, molecular motors 5 , colloids ${ }^{89}$, type II superconducting vortices ${ }^{10} \sqrt{27}$, electrons ${ }^{28}$, active matter ${ }^{29} \sqrt{32}$ and recently in skyrmions 33 . Reversals of motion from the easy to the hard direction may occur as a function of the applied magnetic field or other variables $12|17+19| 40 \mid 43$ due to collective interactions between particles. One of the earliest proposals of a two-dimensional (2D) periodic asymmetric potential that can induce a ratchet effect was the asymmetric funnel geometry 20/44. Wambaugh et al. used a combination of periodic asymmetric funnels and ac driving to produce a net dc motion of type II superconducting vortices. Advanced sample fabrication techniques made it possible to produce this type of vortex ratchet experimentally 21/45/46]. Most of the work on ratchets constructed from asymmetric potentials involves overdamped particles such as colloids or vortices. Recently, however, there has been great interest in the dynamics of skyrmions and proposals to control their motion, and a ratchet effect could be an ideal method for achieving such control.

Magnetic skyrmions are spin textures pointing in all directions and wrapping a sphere in order to form a topologically stable particle-like object ${ }^{47}$ which can be set into motion by the application of a spin polarized current $\frac{48-52}{5}$. In the presence of such external driving, skyrmions can exhibit a depinning threshold similar to that found for superconducting vortices, and it is possible to perform transport measurements and construct skyrmion velocity-force curves $49 \mid 51+54$. Skyrmions show huge promise for applications in spintronics such as racetrack memory devices due to their small size and their ability to be displaced by low currents55-57. In order to realize devices of this type, new methods must be developed which permit the skyrmion motion to be controlled precisely.

A key difference between skyrmions and overdamped particles is that the skyrmion dynamics is dominated by the Magnus term $17 / 49 / 51 / 56 / 58$, which is negligible in other systems. The Magnus term produces a skyrmion velocity contribution that is perpendicular to the applied external drive. It has been proposed that the Magnus force is responsible for the low value of the skyrmion depin-

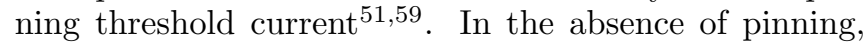
the skyrmion moves at an angle with respect to the driving direction known as the intrinsic skyrmion Hall angle, $\theta_{s k}^{\text {int } 4756 \mid 60}$. This angle depends on the ratio between the Magnus term, $\alpha_{m}$, and the damping term, $\alpha_{d}$. Depending on the system parameters, this ratio can be small or very high 476064 .

The earliest proposal for a skyrmion ratchet 33 involved the same type of quasi-one-dimensional (1D) asymmetric substrate that was known to produce ratcheting of superconducting vortices. For an ac drive applied parallel to the substrate asymmetry direction, the skyrmion exhibits a quantized net displacement, while a Magnusinduced transverse ratchet effect appears when the ac 
drive is applied perpendicular to the substrate asymmetry direction. Further work demonstrated the creation of a vector ratchet with a direction of motion that can be rotated controllably with respect to the orientation of the substrate asymmetry ${ }^{36}$. Recently, a skyrmion ratchet effect produced by a combination of spatial asymmetry and ac driving was used to guide the skyrmion motion in racetrack storage devices 39 . Other skyrmion ratchet effects have been proposed based on currents with a loop structure $^{65}$ or biharmonic in-plane magnetic fields ${ }^{34}$.

Using numerical simulations, we investigate the skyrmion behavior in an asymmetric funnel geometry under applied ac driving at zero temperature. We find that when an ac drive is applied parallel to the funnel axis, a skyrmion ratchet effect occurs in which the skyrmions move with quantized velocities along the easy direction. On the other hand, when the ac drive is applied perpendicular to the funnel axis, there is a Magnus-induced transverse skyrmion ratchet effect in which the skyrmion moves along the hard direction at a constant average velocity. The ability to control the direction of motion of the skyrmion ratchet by varying the ac driving direction can be useful for spintronics applications such as data transfer. To explore this possibility, we simulate a situation in which the skyrmion moves along the easy direction for a period of time, and then switches to motion along the hard direction when the ac driving is rotated by $90^{\circ}$.

\section{SIMULATION}

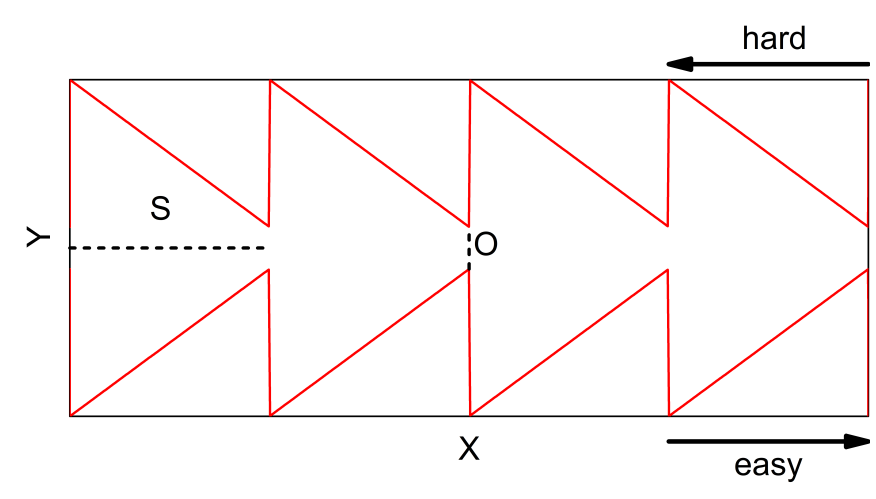

FIG. 1. Illustration of the funnel geometry used in this work, where $S$ is the length of the funnel and $O$ is the width of the funnel opening. In this case the number of funnels $N_{F}=4$, $O=1.0$, and the easy and hard directions of motion are labeled.

We simulate a single skyrmion in an $L \times H$ twodimensional box with periodic boundary conditions in the $x$ direction, as illustrated in Fig. 1. The skyrmion interacts with an asymmetric funnel array aligned with the $x$ direction, where $N_{F}$ is the number of funnels, $S$ is the length of each funnel, and $O$ is the width of the funnel opening. The asymmetry permits "easy" flow along the positive $x$ direction and "hard" flow along the negative $x$ direction. The skyrmion dynamics is governed by the following particle based equation of motion $\frac{59}{}$ :

$$
\alpha_{d} \mathbf{v}+\alpha_{m} \hat{z} \times \mathbf{v}=\mathbf{F}^{W}+\mathbf{F}^{A C}
$$

where $\mathbf{v}$ is the skyrmion velocity. The first term on the left hand side containing the damping constant $\alpha_{d}$ represents the damping originating from the spin precession and dissipation of electrons in the skyrmion core. The second term on the left hand side is the Magnus force, where $\alpha_{m}$ is the Magnus constant. On the right hand side, $\mathbf{F}^{W}$ represents the force exerted by the confining funnel wall. The funnel outline is formed by two asymmetric sawtooth functions and the wall potential is given by a Gaussian form, $U\left(\mathbf{r}_{i w}\right)=U_{0} e^{-\left(\frac{r_{i w}}{a_{0}}\right)^{2}}$, where $\mathbf{r}_{i w}$ is the shortest distance between the skyrmion and the wall, $U_{0}$ is the potential strength, and $a_{0}$ is the wall thickness. The resulting force is $\mathbf{F}^{W}=-\nabla U_{0}=-F_{0} r_{i w} e^{-\left(\frac{r_{i w}}{a_{0}}\right)^{2}} \hat{\mathbf{r}}_{i}$, where $F_{0}=\frac{2 U_{0}}{a_{0}{ }^{2}}$. The term $\mathbf{F}^{A C}$ represents the applied ac drive, $\mathbf{F}^{A C}=A \sin (2 \pi \omega t) \hat{\mathbf{x}}+B \cos (2 \pi \omega t) \hat{\mathbf{y}}$, where $\omega$ is the frequency of the ac drive and $A$ and $B$ are the ac drive amplitudes in the $x$ and $y$ directions, respectively. For each value of $A$ or $B$ we perform a simulation spanning $5 \times 10^{6}$ time steps, with a step size of $10^{-3}$, in order to ensure the system has reached a steady state. We measure the average velocities $\left\langle V_{x}\right\rangle=2 \pi\langle\mathbf{v} \cdot \widehat{\mathbf{x}}\rangle / \omega S$ and $\left\langle V_{y}\right\rangle=2 \pi\langle\mathbf{v} \cdot \widehat{\mathbf{y}}\rangle / \omega S$, where $S$ is the length of a single funnel. The value of $A$ or $B$ is incremented by $\delta A(B)=0.005$. In this work we fix $U_{0}=1.0$, $\omega=2 \times 10^{-5}$, the sample length $L=20.0$, the sample height $H=8.0$, and $a_{0}=0.02$. We normalize all distances by the screening length $\xi$ and select the damping and Magnus constants such that $\alpha_{m}{ }^{2}+\alpha_{d}^{2}=1$.

\section{AC DRIVE IN THE x DIRECTION}

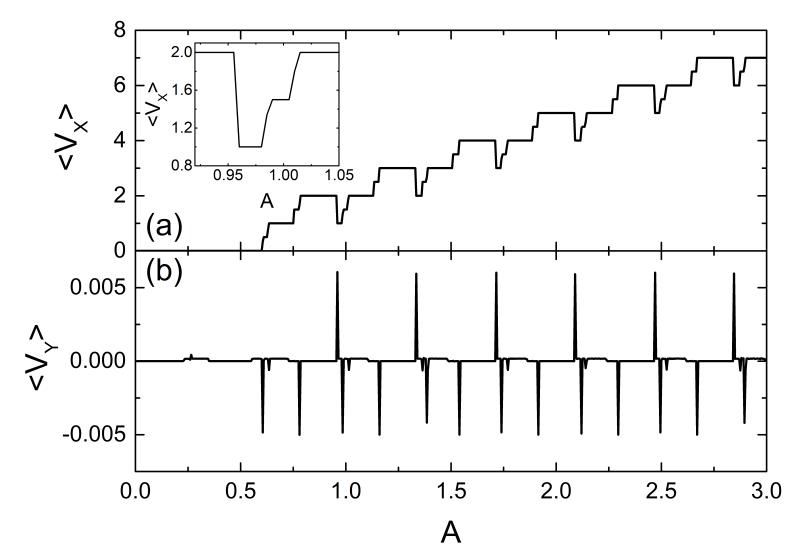

FIG. 2. (a) $\left\langle V_{x}\right\rangle$ and (b) $\left\langle V_{y}\right\rangle$ versus ac drive amplitude $A$ for a sample with $\alpha_{m} / \alpha_{d}=0.5, N_{F}=4$, and $O=1.0$. Here the ac driving is only along the $x$ direction. The inset of (a) shows a blowup of panel (a) over the range $0.92<A<1.05$. 
We first consider an ac drive applied parallel to the funnel array axis, where $A \neq 0$ and $B=0$. In Fig. 2 we plot $\left\langle V_{x}\right\rangle$ and $\left\langle V_{y}\right\rangle$ as a function of the ac drive amplitude $A$ for a system with $\alpha_{m} / \alpha_{d}=0.5$. The average $x$ velocity $\left\langle V_{x}\right\rangle$, shown in Fig. 2(a), has a step-like behavior superimposed on a monotonic increase. Here the combination of the ac drive and the broken spatial symmetry of the funnel array generates a ratchet effect in which there is a net dc motion of the skyrmion in the $+x$ direction. Although $\left\langle V_{y}\right\rangle$ in Fig. 2(b) is very close to zero, there is a spike of transverse motion at the edge of every step in $\left\langle V_{x}\right\rangle$.
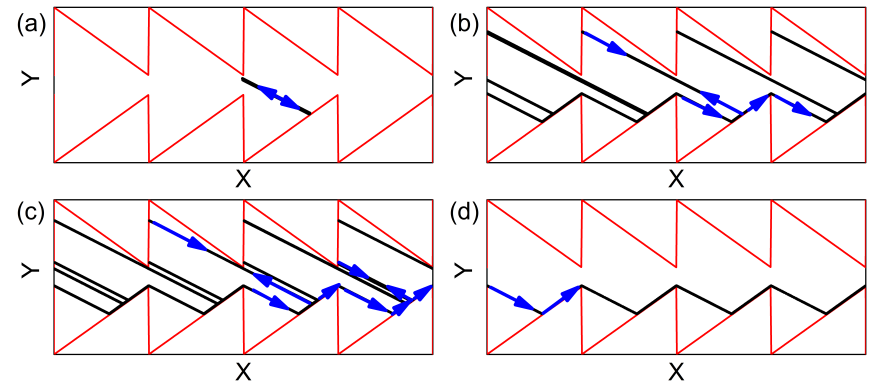

FIG. 3. Funnel wall (red lines) and the skyrmion trajectory (black lines) for a single skyrmion interacting with a funnel array with $N_{F}=4, O=1.0, S=5, \alpha_{m} / \alpha_{d}=0.5$, and an ac drive applied along the $x$ axis with $B=0$. Blue arrows indicate the direction of skyrmion motion. (a) At $A=0.25$, the skyrmion forms a closed orbit inside a single funnel. (b) At $A=0.97$, there is a translating orbit with a net dc motion along the $+x$ direction. On average the skyrmion translates by a distance of $1.0 S$ per ac drive cycle. (c) At $A=1.76$, the skyrmion translates an average of $3.5 S$ per cycle. (d) At $A=2.4$ the skyrmion translates an average of $6 S$ per cycle.

In Fig. 3 we illustrate some representative skyrmion trajectories for the system in Fig. 2. At $A=0.25$, Fig. 3(a) shows that the ac drive amplitude is not large enough to generate a ratchet motion and the skyrmion forms a localized orbit inside one of the funnels. For $A=0.97$ in Fig. 3(b), we find $+x$ direction skyrmion ratchet motion in which the skyrmion translates by an average distance of $1.0 S$ during each ac drive cycle. The trajectory trace indicates that the skyrmion moves forward by $2 S$ during the $+x$ portion of the ac drive cycle, and backward by $1.0 S$ during the $-x$ portion of the drive cycle. In Fig. 3. (c) at $A=1.76$, the skyrmion translates an average of $3.5 S$ per cycle, while at $A=2.4$ in Fig. 33(d), the skyrmion flows faster at a rate of $6 S$ per cycle, always in the $+x$ direction.

Since the ratchet effect requires asymmetry in the potential, we expect that changes to the funnel shape or density will alter the skyrmion behavior. In Fig. 4(a) we show the results of changing the width $O$ of the funnel opening while holding the number of funnels fixed at $N_{F}=4$, while in Fig. 4 (b) we vary $N_{F}$ and fix $O=0.28$. Figure 4(a) shows that when the funnel opening width $O$ is reduced, the onset of the ratchet effect shifts to lower values of $A$. This is due to a reduction in the probability

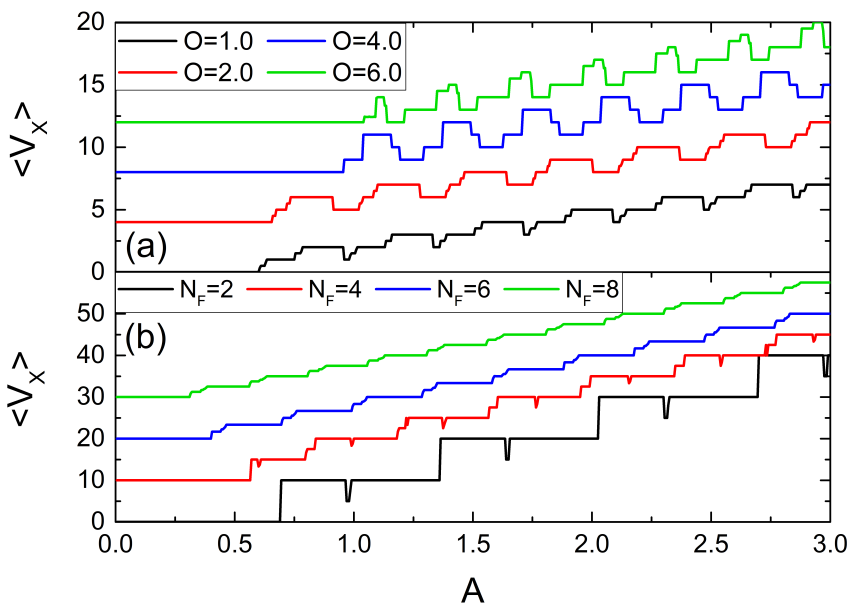

FIG. 4. Plots of $\left\langle V_{x}\right\rangle$ vs $A$ in samples with $\alpha_{m} / \alpha_{d}=0.5$. (a) Varied funnel opening widths $O$ for a fixed number of funnels $N_{F}=4$. (b) Varied $N_{F}$ for fixed $O=0.28$. For clarity, the curves have been offset vertically. To facilitate comparison, all velocities are normalized using $S=5$ even though $S$ varies when $N_{F}$ is changed.

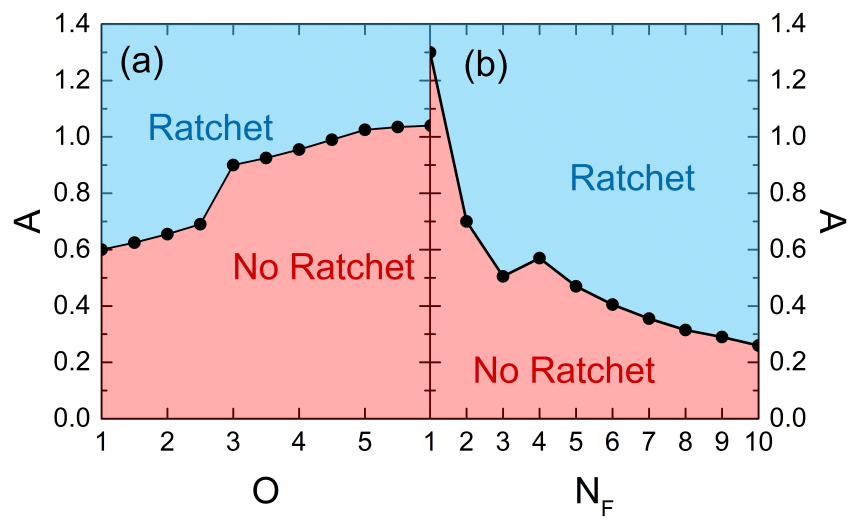

FIG. 5. Dynamic phase diagram as a function of ac drive amplitude $A$ vs (a) $O$ for $N_{F}=4$ and (b) $N_{F}$ for $O=0.28$ in a system with $\alpha_{m} / \alpha_{d}=0.5$. Red regions exhibit no ratchet effect, while blue regions indicate the appearance of ratcheting in the $+x$ direction.

that the skyrmion will be able to pass backwards through the funnel during the $-x$ portion of the drive cycle as the funnel opening becomes narrower. We also find that the shape of the $\left\langle V_{x}\right\rangle$ signal is modified as $O$ is varied. In Fig. 4(b), where $O$ is fixed to $O=0.28$, the ratcheting effect is enhanced as the number of funnels $N_{F}$ increases. Here, the length $S$ of each funnel diminishes as $N_{F}$ becomes larger since the width of the sample is held constant. As a result, the distance the skyrmion must travel to move from one funnel plaquette to the next decreases with increasing $N_{F}$, and the amplitude $A$ at which the ratchet effect first appears shifts to lower values. To summarize the influence of the funnel shape on the skyrmion behavior, we plot dynamic phase diagrams as a function of ac drive amplitude $A$ versus $O$ in Fig. 5(a) and ver- 
sus $N_{F}$ in Fig. 5(b). Figure 5(a) shows that the onset of ratchet motion drops to smaller $A$ as the funnel opening width $O$ is reduced, while in Fig. 5(b), the threshold amplitude for ratchet motion generally diminishes as the number of funnels increases.

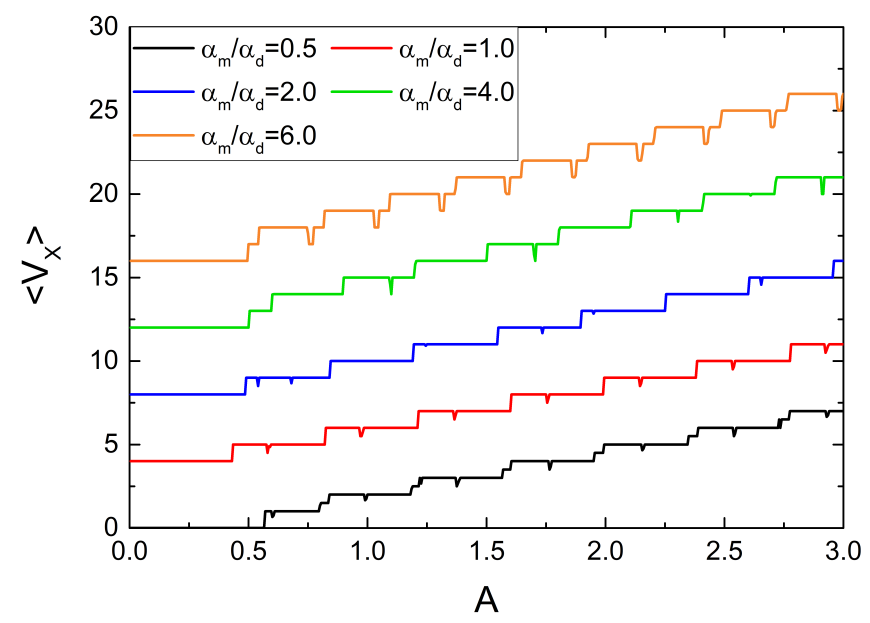

FIG. 6. $\left\langle V_{x}\right\rangle$ vs $A$ for different values of $\alpha_{m} / \alpha_{d}$ in samples with $O=0.28$ and $N_{F}=4$. The curves have been offset vertically for clarity.

To explore the effect of the Magnus term intensity on the dynamics, in Fig. 6 we plot $\left\langle V_{x}\right\rangle$ versus $A$ at different values of $\alpha_{m} / \alpha_{d}$ ranging from $\alpha_{m} / \alpha_{d}=0.5$ to $\alpha_{m} / \alpha_{d}=$ 6.0 for a system with $O=0.28$ and $N_{F}=4$. As the Magnus term becomes stronger, the skyrmion velocity steps become narrower, while there is little change in the depinning threshold. In previous work on skyrmion ratchets in quasi-1D asymmetric substrates ${ }^{33}$, when the ac drive was applied parallel to the asymmetric potential, increasing the Magnus term decreased the range of ac driving forces over which the ratchet motion appeared. For the funnel geometry considered here, we find only a weak impact of the Magnus term on the magnitude of the ratcheting for ac driving applied parallel to the funnel asymmetry direction.

\section{AC DRIVE IN THE y DIRECTION}

We next consider an ac drive applied perpendicular to the funnel axis, with finite $B$ and $A=0$. In Fig. 7 we show results from a system with $N_{F}=4, O=1.0$, and $\alpha_{m} / \alpha_{d}=0.5$. For $B>0.595$, a Magnus-induced transverse ratchet occurs and the skyrmion flows in the $-x$ direction, as indicated in Fig. 77(a). Note that the motion maintains a steady average velocity of $\left\langle V_{x}\right\rangle=-1$ over a wide range of applied ac drive amplitudes. The stability in this motion is of interest for technological applications since the translation speed remains the same for various values of ac amplitudes. In Fig. 7(b) we illustrate the localized skyrmion orbit at $B=0.5$, where the skyrmion remains trapped inside a single funnel. In
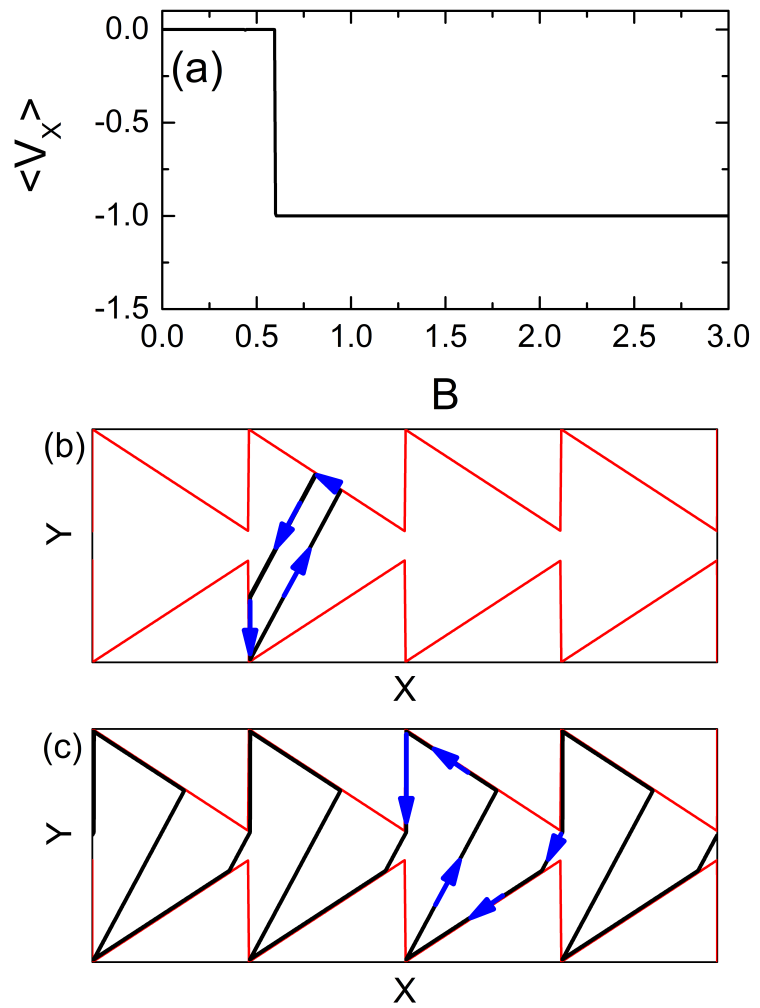

FIG. 7. A system with $N_{F}=4, O=1.0, S=5$, $\alpha_{m} / \alpha_{d}=0.5$, and an ac drive applied along the $y$ axis with $A=0$. (a) $\left\langle V_{x}\right\rangle$ vs ac drive amplitude $B$. For $B>0.595$, ratcheting motion in the $-x$ direction appears. (b,c) Funnel wall (red lines) and the skyrmion trajectory (black lines) for the same system. Blue arrows indicate the direction of skyrmion motion. (b) At $B=0.5$, the skyrmion forms a stationary orbit localized inside a single funnel. (c) Ratcheting motion in the $-x$ direction at $B=1.0$, where the average velocity is $-1.0 S$ per cycle.

Fig. 7(c) we show a skyrmion ratchet trajectory, where the funnel walls guide the skyrmion motion in the $-x$ direction.

In Fig. 8 we show the effect of varying $O$ and $N_{F}$ on the net ratcheting motion. For samples in which the number of funnels is fixed at $N_{F}=4$ and $O$ is varied over the range $O=1$ to $O=6$, the plot of $\left\langle V_{x}\right\rangle$ versus $B$ in Fig. 8(a) indicates that there is only a small upward shift in the depinning threshold as the funnel opening becomes wider. There is a single sharp transition to motion for small $O$, but at larger $O$ multiple dynamical phases appear and produce numerous steps in the velocity-force curve. A variety of skyrmion orbits appear when the funnel openings are wide, as illustrated in Fig. 9 (a) at $B=1.0$. For all values of $O$, there is a critical ac drive amplitude above which the motion saturates into a state with $\left\langle V_{x}\right\rangle=-1.0$, as shown in Fig. 9(b) at $B=1.5$. When the number of funnels is increased for fixed $O=0.28$, as in Fig. 8(b), the depinning force decreases since the skyrmion does not need to move as far to escape from one funnel into an adjacent funnel. For 


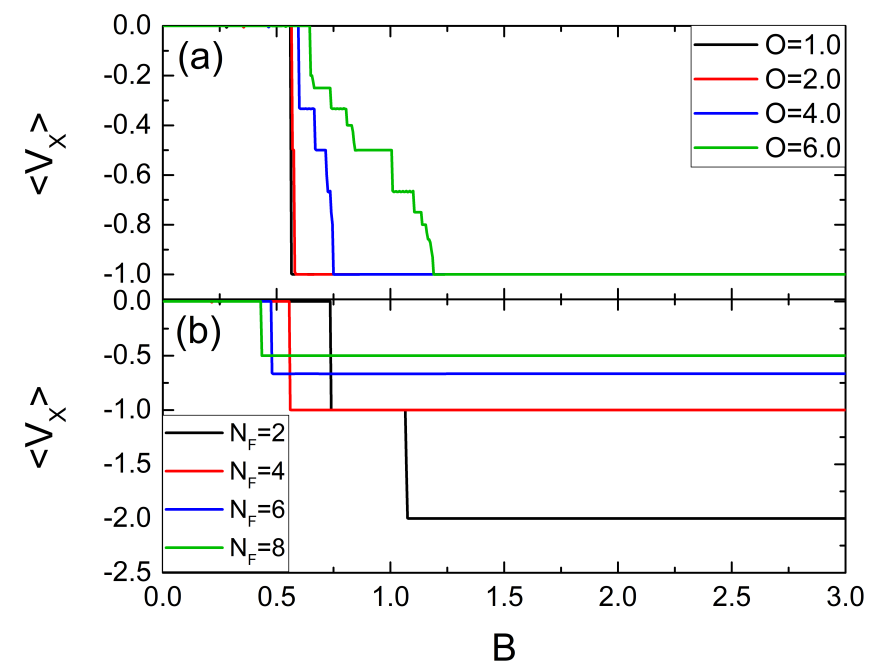

FIG. 8. $\left\langle V_{x}\right\rangle$ vs $B$ for samples with $\alpha_{m} / \alpha_{d}=0.5$ and $y$ direction ac driving with $A=0$. (a) Varied funnel opening widths $O$ and fixed $N_{F}=4$. (b) Varied $N_{F}$ and fixed $O=$ 0.28 . To facilitate comparison, all velocities are normalized using $S=5$ even though $S$ varies when $N_{F}$ is changed.
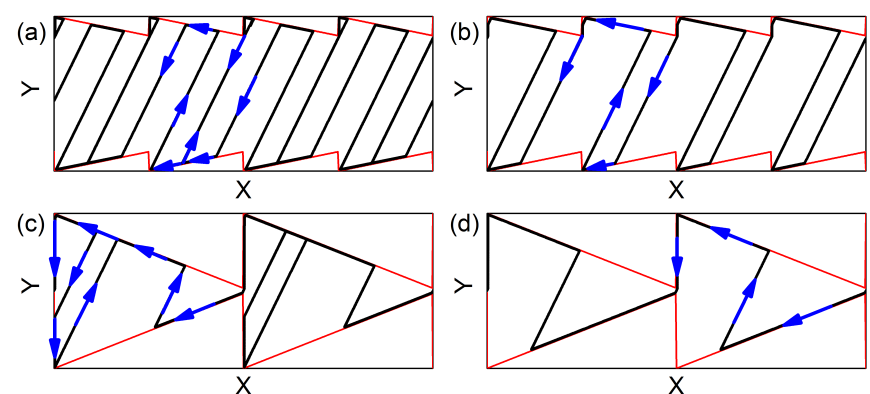

FIG. 9. Funnel wall (red lines) and the skyrmion trajectory (black lines) in a system with $\alpha_{m} / \alpha_{d}=0.5$ and an ac drive applied along the $y$ axis with $A=0$, where a net dc motion along $-x$ appears. In panels (a) and (b), $N_{F}=4$ and $O=6.0$, while in panels (c) and (d), $N_{F}=2$ and $0=0.28$. (a) A complex intermediate phase at $B=1.0$. (b) The saturated stable translating orbit at $B=1.5$. (c) At $B=1.0$ the net skyrmion speed is reduced. (d) At $B=1.5$, the skyrmion moves more rapidly with $\left\langle V_{x}\right\rangle=-2.0$.

this value of $O$, there is only a single translating state except when $N_{F}=2$. The larger amount of space inside each funnel for the $N_{F}=2$ system permits the formation of two different translating motions, as illustrated in Fig. 9(c,d) at $B=1.0$ and $B=1.5$, respectively.

We summarize the skyrmion behavior under $y$ direction ac driving in Fig. 10 where we plot a dynamic phase diagram as a function of $B$ versus $O$ for $N_{F}=4$ in Fig. 10(a) and as a function of $B$ versus $N_{F}$ for $O=0.28$ in Fig. 10(b). The width of the funnel opening has little effect on the onset of the ratcheting motion, as shown in Fig. $10(\mathrm{a})$, since the skyrmion flow is along the $-x$ direction. As the number of funnels increases, Fig. 10(b) indicates that the onset of ratcheting motion drops to

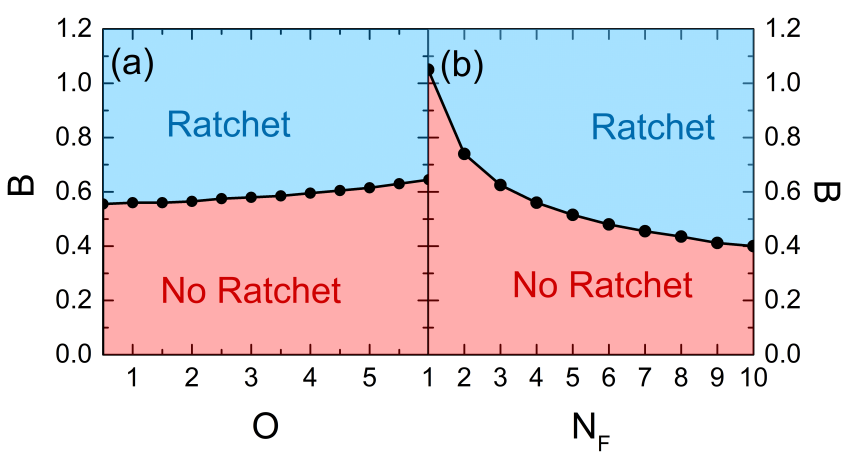

FIG. 10. Dynamic phase diagram as a function of ac drive amplitude $B$ vs (a) $O$ for $N_{F}=4$ and (b) $N_{F}$ for $O=0.28$ in a system with $\alpha_{m} / \alpha_{d}=0.5$ and $y$ direction ac driving with $A=0$. Red regions exhibit no ratchet effect, while blue regions indicate the appearance of ratcheting in the $-x$ direction.

lower values of $B$.

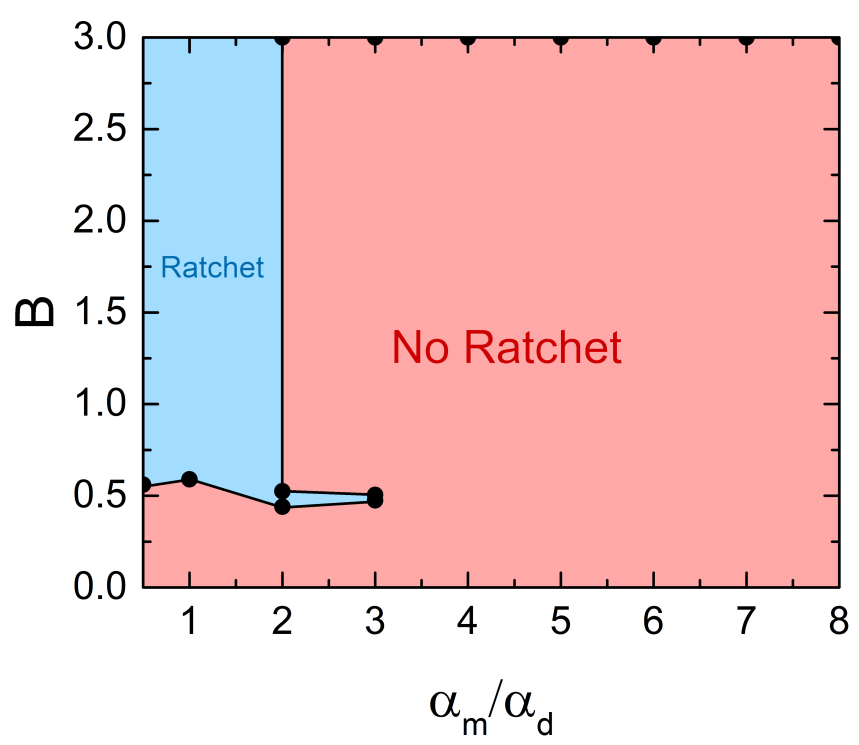

FIG. 11. Dynamic phase diagram as a function of $B$ vs $\alpha_{m} / \alpha_{d}$ for samples with $O=0.28$ and $N_{F}=4$ under $y$ direction ac driving with $A=0$. Red regions exhibit no ratchet effect, while blue regions indicate the appearance of ratcheting in the $-x$ direction.

In Fig. 11, we plot a dynamic phase diagram as a function of $B$ versus $\alpha_{m} / \alpha_{d}$ for samples with $O=0.28$ and $N_{F}=4$ to show how the size of the Magnus term impacts the transverse ratchet effect. When $\alpha_{m} / \alpha_{d} \geq 3.0$, no ratcheting motion appears. The reduction of a Magnusinduced transverse ratchet as a function of increasing Magnus force was observed previously in systems with quasi-1D substrates 33 . For our funnel geometry, although Fig. 6 showed that $\alpha_{m} / \alpha_{d}$ has little impact on the ratchet effect when the ac drive is applied parallel to the $x$ direction, Fig. 11 indicates that for ac driving applied parallel to the $y$ direction, modifications to the Magnus 
term can strongly modify the ratcheting behavior. For $\alpha_{m} / \alpha_{d}=2.0$ and 3.0, we observe a reentrant pinning phase with a small window of ratcheting motion near $B=0.5$.

\section{BIHARMONIC AC DRIVING}

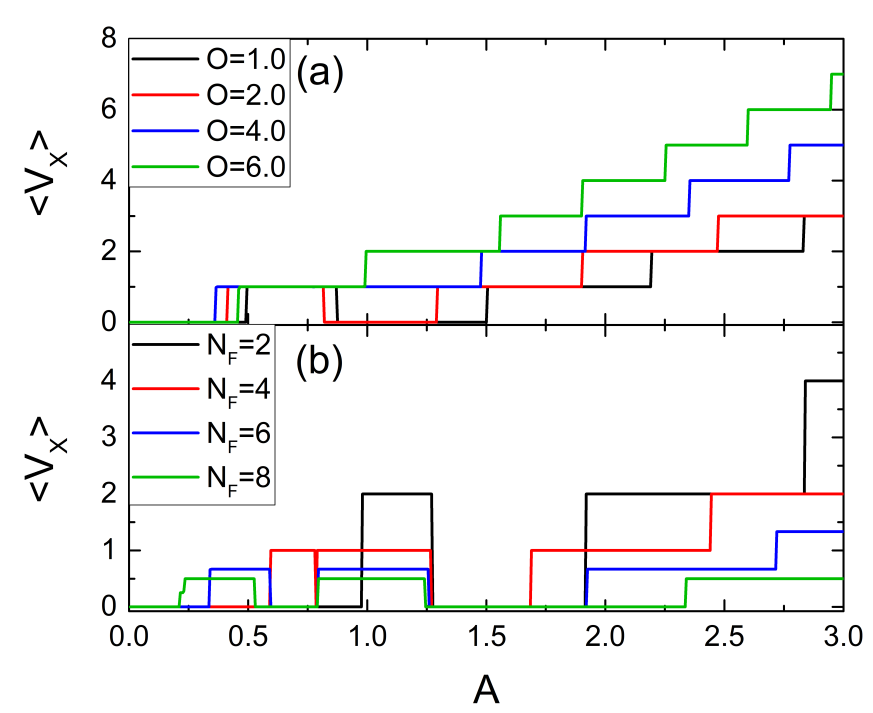

FIG. 12. $\left\langle V_{x}\right\rangle$ vs $A$ for samples with biharmonic driving in which $A=B$ and $\alpha_{m} / \alpha_{d}=0.5$. (a) Varied $O$ and fixed $N_{F}=4$. (b) Varied $N_{F}$ and fixed $O=0.28$. To facilitate comparison, all velocities are normalized using $S=5$ even though $S$ varies when $N_{F}$ is changed.
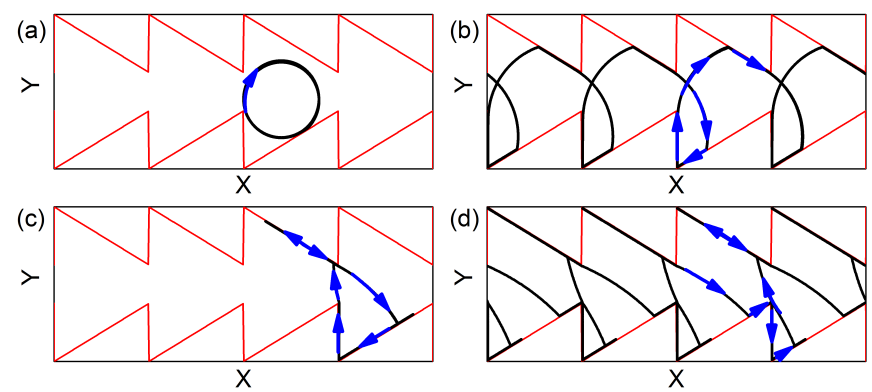

FIG. 13. Funnel wall (red lines) and the skyrmion trajectory (black lines) in a system with $\alpha_{m} / \alpha_{d}=0.5$ and a biharmonic ac drive applied in both the $x$ and $y$ directions with $B=A$, $N_{F}=4$ and $O=2.0$. (a) At $A=0.25$, a localized orbit forms and there is no net dc motion. (b) At $A=0.5$ the skyrmion translates in the $+x$ direction. (c) At $A=1.0$, a complex localized orbit forms between two adjacent funnels in the reentrant pinning phase. (d) At $A=1.5$, above the reentrant pinning window, the skyrmion has a net motion in the $+x$ direction.

We next consider biharmonic ac driving where both $A$ and $B$ are finite and the skyrmion is simultaneously driven in both the $x$ and $y$ directions. In Fig. 12 we plot $\left\langle V_{x}\right\rangle$ versus $A$ for samples with $\alpha_{m} / \alpha_{d}=0.5$ and $B=A$. For a fixed number of funnels $N_{F}=4$ and varied $O$, as in Fig. 12(a), a reentrant pinning phase appears for $O \leq 2.0$ in which the net dc motion disappears. For higher values of $O$, this reentrant pinning phase vanishes since multiple trapped orbits can no longer form inside individual funnels. In Fig. 12(b), where we fix $O=0.28$ and vary $N_{F}$, we are in a regime where the reentrant pinning phase is always present, and the range of ac drive amplitudes over which the reentrant pinning appears changes with $N_{F}$. For large $N_{F}$, such as $N_{F}=6$ and $N_{F}=8$, a second reentrant pinning phase emerges. Here, the shorter funnel lengths at high $N_{F}$ make it possible for additional trapped nontranslating skyrmion orbits to form. In Fig. 13 we show representative skyrmion trajectories for the system from Fig. 12 with $N_{F}=4$ and $O=2.0$. At $A=0.25$ in Fig. 13(a), there is no net dc motion since the drive amplitude is not large enough for a delocalized orbit to form. In contrast, at $A=0.5$ in Fig. 13(b), we find a translating orbit with motion in the $+x$ direction. In Fig. 13(c) we illustrate the reentrant pinning phase at $A=1.0$ where a localized orbit spanning two funnels appears, while in Fig. 13(d) at $A=1.5$, we find a translating orbit with a net motion in the $+x$ direction above the reentrant pinning regime.

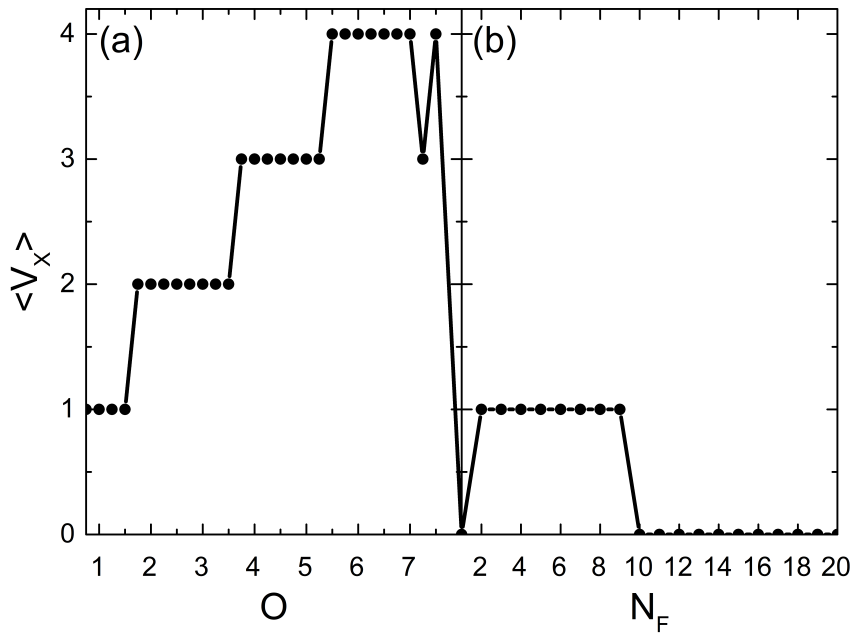

FIG. 14. $\left\langle V_{x}\right\rangle$ vs (a) $O$ for $N_{F}=4$ and (b) $N_{F}$ for $O=1.0$ in samples with biharmonic driving, $\alpha_{m} / \alpha_{d}=0.5$, and $A=$ $B=2.0$. Due to the normalization, $\left\langle V_{x}\right\rangle$ provides a measure of the number of funnel plaquettes spanned by the translating trajectory during each ac drive cycle.

When its value is quantized, $\left\langle V_{x}\right\rangle$ provides a measurement of the number of funnel plaquettes spanned by a translating trajectory during each ac drive cycle. We can use this measure to explore the effects of varying the funnel parameters out to their extreme values. In Fig. 14(a) we plot $\left\langle V_{x}\right\rangle$ versus $O$ under biharmonic driving with $A=B=2.0$ at $N_{F}=4$, where we find a clear increase in the skyrmion velocity as the funnel opening becomes larger. The ratchet effect increases with increasing $O$ due to the increased probability for the skyrmion to reach the 
next funnel along the easy direction. When $O$ reaches its maximum size of $O=8.0$, however, the funnel structure vanishes and thus the skyrmion ratchet effect also disappears. Abrupt vanishing of a ratchet effect with funnel aperture is common in funnel ratchet systems. When we fix $O=1.0$ and vary $N_{F}$, Fig. 14(b) shows that the number of funnels visited decreases with increasing $N_{F}$ since the funnel walls become steeper as each individual funnel becomes shorter. When $N_{F}>10$, the skyrmion ratchet is lost for this choice of driving amplitude, $A=B=2.0$.

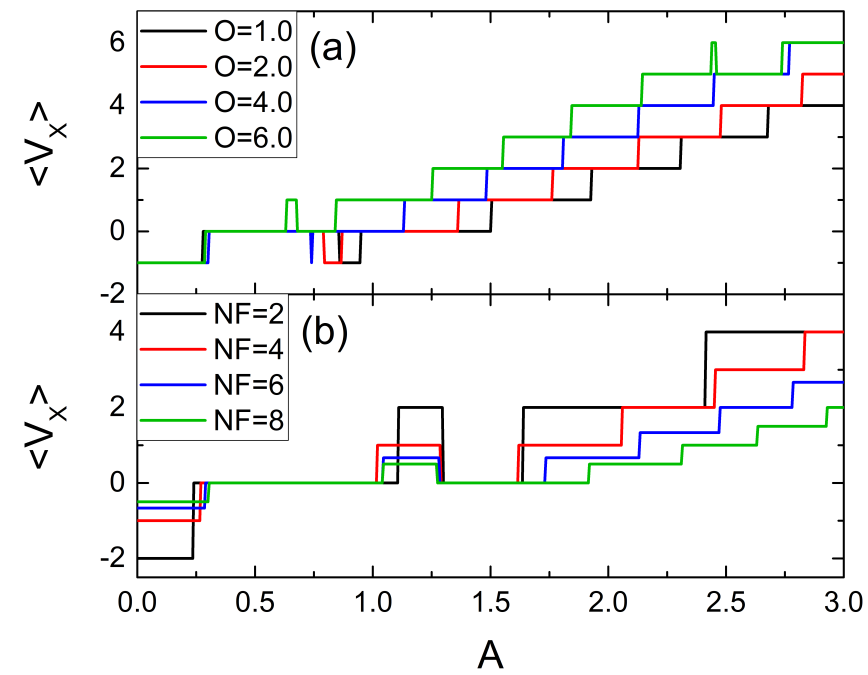

FIG. 15. $\left\langle V_{x}\right\rangle$ vs $A$ in samples with biharmonic driving and fixed $B=1.5$ at $\alpha_{m} / \alpha_{d}=0.5$. (a) Varied $O$ for fixed $N_{F}=4$. (b) Varied $N_{F}$ for fixed $O=0.28$. To facilitate comparison, all velocities are normalized using $S=5$ even though $S$ varies when $N_{F}$ is changed.
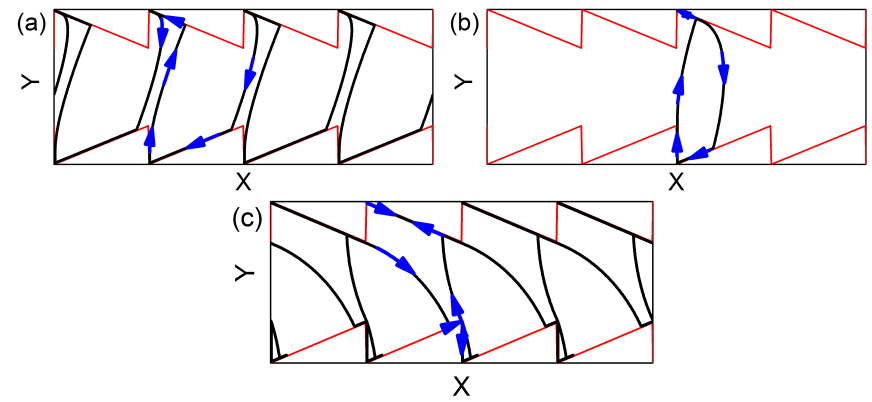

FIG. 16. Funnel wall (red lines) and the skyrmion trajectory (black lines) in a system with $\alpha_{m} / \alpha_{d}=0.5$ and a biharmonic ac drive with $B=1.5, N_{F}=4$, and $O=4.0$. (a) At $A=$ 0.25 , the skyrmion translates in the $-x$ direction due to the Magnus-induced transverse ratchet effect. (b) At $A=0.5$, there is an intermediate state with no dc motion. (c) At $A=1.2$, the skyrmion translates in the $+x$ direction due to the parallel ratchet effect.

In Fig. 15 we plot $\left\langle V_{x}\right\rangle$ versus $A$ for a biharmonic ac drive system with fixed $B=1.5$ at $\alpha_{m} / \alpha_{d}=0.5$. An ac drive of this type causes a free skyrmion to execute an asymmetric orbit, and this asymmetry enhances

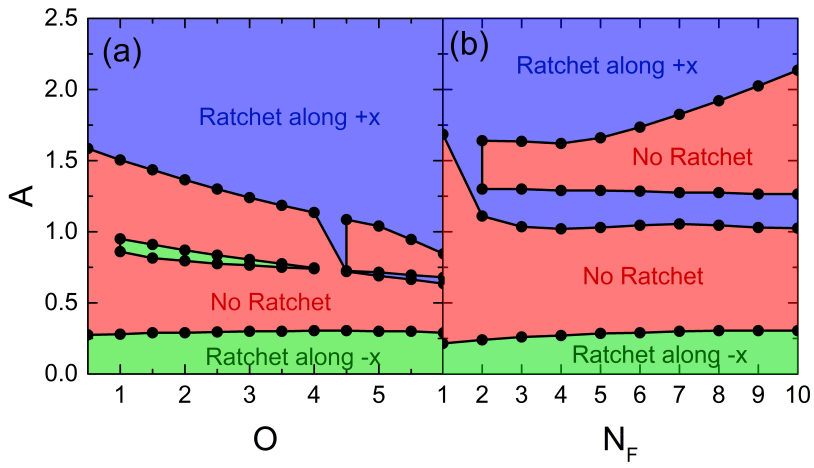

FIG. 17. Dynamic phase diagrams for samples with biharmonic driving, $B=1.5$, and $\alpha_{m} / \alpha_{d}=0.5$ as a function of (a) $A$ vs $O$ with $N_{F}=4$ and (b) $A$ vs $N_{F}$ with $O=0.28$. Green areas indicate ratcheting motion in the hard or $-x$ direction, purple areas have ratcheting motion in the easy or $+x$ direction, and red areas show no ratchet effect.

the ratchet effect. In the funnel geometry, the resulting skyrmion behavior consists of a combination of the effects found for unidirectional ac drives. For very low values of $A$, the skyrmion moves in the $-x$ direction due to the strong Magnus-induced transverse ratchet which appears at $B=1.5$, as illustrated in Fig. 16(a) at $A=0.25$. For higher values of $A$, the parallel ratchet mechanism becomes dominant and the skyrmion moves in the $+x$ direction, as shown in Fig. 16(c) at $A=1.2$. An intermediate state appears between these regimes when the Magnusinduced transverse ratchet and the parallel ratchet cancel each other, producing a localized orbit with no dc motion, as shown in Fig. 16.(b) at $A=0.5$.

In Fig. 17(a) we plot a dynamic phase diagram as a function of $A$ versus $O$ for a biharmonic system with $B=1.5, \alpha_{m} / \alpha_{d}=0.5$, and $N_{F}=4$, while in Fig. 17(b) we show a similar dynamic phase diagram as a function of $A$ versus $N_{F}$ for the same system at fixed $O=0.28$. We find a reversal of the ratchet direction from $-x$ to $+x$ as $A$ increases, along with multiple reentrant pinning regions in which ratcheting behavior does not occur. Figure 17 (a) shows a window of $-x$ direction ratcheting over the range $1.0<O<4.0$ in the middle of the pinned region when $A \approx 0.8$. A similar window of $+x$ direction ratcheting appears in the same region of $A$ for $O>4.5$. In Fig. 17(b), we find a pinned phase for $N_{F} \geq 2$ close to $A=1.3$ where the $-x$ ratchet motion ceases, followed by the emergence of ratcheting motion in the $+x$ direction for higher $A$. Judicious selection of both $O$ and $N_{F}$ determines the number and type of ratcheting phases that can be accessed as $A$ is varied.

\section{GUIDED MOTION USING AC DRIVES}

In the previous section, we showed that a skyrmion can be set into motion in the easy $(+x)$ or hard $(-x)$ direction of the funnel axis depending on the orientation 

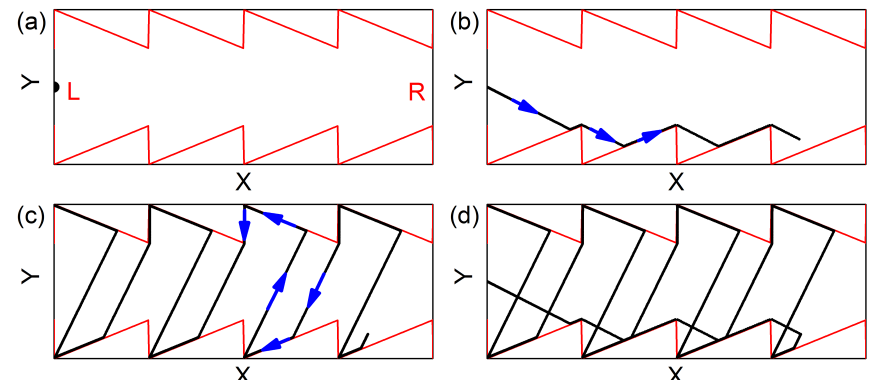

FIG. 18. Funnel wall (red lines) and the skyrmion trajectory (black lines) under biharmonic driving in a system with $N_{F}=$ $4, O=4.0$, and $\alpha_{m} / \alpha_{d}=0.5$ illustrating trajectory guiding from the left funnel, marked L, to the right funnel, marked $\mathrm{R}$, and back. (a) Skyrmion starting position in funnel $\mathrm{L}$ at $t=0$. (b) First stage of operation with $A=2.0$ and $B=0.0$, where the skyrmion moves from $L$ to $R$. (c) Second stage of operation with $A=0.0$ and $B=2.0$, where the skyrmion moves from $R$ back to $L$. (d) The complete trajectory.

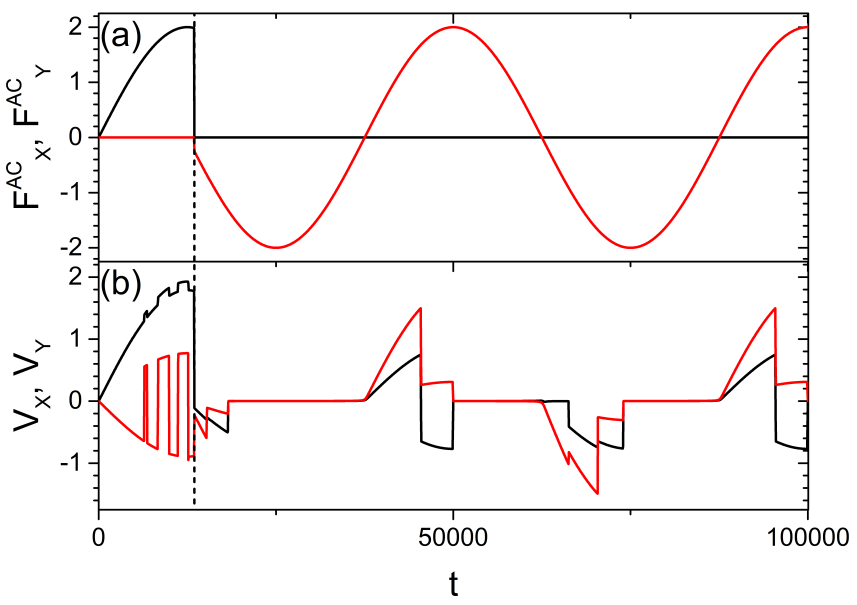

FIG. 19. (a) The applied ac drive forces, $F_{x}^{A C}$ (black) and $F_{y}^{A C}$ (red), versus time $t$ for the guided skyrmion motion in Fig. 17. The time axis is truncated at $t=100,000$, but the same ac driving is applied at later times to achieve the transport in Fig. 17. (b) The instantaneous skyrmion velocities $V_{x}$ (black) and $V_{y}$ (red) versus $t$ under the driving shown in panel (a). The vertical dashed line indicates the time at which the ac drive direction is changed.

of the ac driving force. We now consider a situation in which a skyrmion must be guided from an initial funnel to another funnel and must then return to its original position under only ac driving. This process is relevant to spintronics devices in which a skyrmion acting as an information carrier is guided through the sample in order to transmit information. In Fig. 18(a) we illustrate the sample in which the skyrmion will move from the left funnel labeled $\mathrm{L}$ to the right funnel labeled $\mathrm{R}$ and back again. In Fig. 19. a) we plot the applied ac drive signals $F_{x}^{A C}$ and $F_{y}^{A C}$ used to produce this motion as a function of time, and in Fig. 19(b) we show the corresponding instantaneous skyrmion velocities $V_{x}$ and $V_{y}$. In order to guide the skyrmion along the easy direction towards funnel $R$, we apply ac driving in the $x$ direction with $A=2$ and $B=0$ for 13500 time steps. The skyrmion trajectory for this interval is illustrated in Fig. 18(b). As shown in Fig. 19, only $1 / 4$ of an ac drive cycle is sufficient to bring the skyrmion to funnel $R$ due to the high efficiency of motion in the easy direction. During this time interval, Fig. 19 (b) indicates that the skyrmion velocity $V_{x}$ is always positive. In order to guide the skyrmion back to funnel $L$, we switch the ac driving into the $y$ direction with $A=0$ and $B=2$ in order to produce a Magnus-induced transverse ratchet effect that translates the skyrmion in the $-x$ direction. The skyrmion trajectory for this interval of time, which extends to $t=200000$, is illustrated in Fig. 18(c). The driving in this second stage of motion must be applied for a much longer time interval since the motion in the hard direction is relatively inefficient compared to motion in the easy direction. This is highlighted in Fig. 19(b) where the skyrmion velocity $V_{x}$ drops to zero multiple times during the motion in the hard direction back towards funnel L. We even observe time intervals in which the skyrmion moves in the $+x$ direction, away from funnel L, before reversing direction and moving back toward funnel L. The complete skyrmion trajectory is shown in Fig. 18(d). We expect that similar results could be achieved using asymmetric ac drives in which neither $A$ nor $B$ is zero. As mentioned in Section $\mathrm{V}$, for a fixed $B$, the skyrmion moves in the $-x$ direction for low values of $A$ and in the $+x$ direction for high values of $A$. If asymmetric ac drives of this type were used to achieve the type of transport illustrated in Fig. 18, the trajectories would be more complex.

\section{DISCUSSION}

Using a combination of funnel geometries and ac driving, it is possible to control the skyrmion direction of motion accurately. When the ac drive is applied parallel to the funnel axis, we find easy or $+x$ direction ratcheting, while ratcheting in the hard or $-x$ direction occurs for an ac drive applied perpendicular to the funnel axis. The ratchet motion in the easy direction of the funnel has been observed in a wide variety of systems

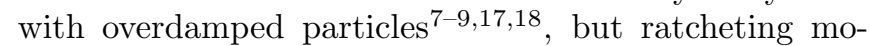
tion in the hard direction appears only for particles with strong Magnus terms 33 . This feature can be exploited to build devices in which the direction of the skyrmion transport must be controlled precisely. A future interesting aspect to explore is the influence of temperature. It is well-known that thermal effects can modify phase transition points and in some cases can completely destroy

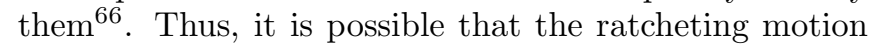
along the hard direction could be destroyed by strong thermal fluctuations, since our results indicate that this motion is not very efficient. Our simulation employs a particle-based skyrmion model 59 ; however, skyrmions also have internal structure that can be excited by ap- 
plied ac drives, or that can be deformed by an interaction with a wall barrier ${ }^{51}$. Such effects could be further explored in continuum-based simulations. We considered the dynamics of a single skyrmion, but we expect our results to be general for the case of multiple skyrmions provided the skyrmion density is sufficiently low. For multiple interacting skyrmions at higher densities, the skyrmion-skyrmion interactions cannot be neglected and could produce significant modifications of the dynamics due to collective effects $67 / 68$. Our results should be applicable not only to skyrmions, but to other magnetic textures exhibiting significant Magnus effects, such as merons 69 .

\section{SUMMARY}

In this work we investigated the skyrmion behavior at zero temperature in a funnel geometry under applied ac driving. We show that for ac driving applied either parallel or perpendicular to the funnel axis, the skyrmion can undergo net dc transport along either the easy or the hard direction of the funnel. For ac driving applied parallel to the funnel axis, the skyrmion moves in the easy direction with a quantized velocity that increases monotonically with increasing ac drive amplitude. In contrast, when the ac driving is applied perpendicular to the funnel axis, the skyrmion flows in the hard direction at a constant average velocity due to a Magnus-induced transverse ratchet effect. Increasing the relative importance of the Magnus term compared to the damping term only weakly affects the easy direction ratcheting, but can destroy the dc motion of the transverse ratchet effect. For biharmonic driving with equal drive amplitudes both parallel and perpendicular to the funnel axis, a reentrant pinning phase appears that depends on the size of the funnel opening and the number of funnels. Under asymmetric biharmonic ac driving, where the perpendicular ac drive amplitude is held fixed and the parallel ac drive amplitude $A$ is varied, we find a combination of the effects observed for separate ac driving along each axis. For low values of $A$, the skyrmion moves along the hard direction due to the prominent transverse ratchet effect; however, for higher values of $A$, a transition occurs to motion along the easy direction due to the strengthening of the parallel ratchet effect. These results may be useful for future devices since they indicate that the skyrmion motion in a funnel geometry can be controlled using only ac driving. To illustrate this, we demonstrate that a skyrmion can be transported to a series of predetermined locations by varying the ac driving over time, simulating a data transfer process.

\section{ACKNOWLEDGMENTS}

This work was supported by the US Department of Energy through the Los Alamos National Laboratory. Los Alamos National Laboratory is operated by Triad National Security, LLC, for the National Nuclear Security Administration of the U. S. Department of Energy (Contract No. 892333218NCA000001). N.P.V. acknowledges funding from Fundação de Amparo à Pesquisa do Estado de São Paulo - FAPESP (Grant 2017/20976-3).
1 P Reimann, "Brownian motors: noisy transport far from equilibrium," Phys. Rep. 361, 57-265 (2002)

2 R. D. Vale and F. Oosawa, "Protein motors and Maxwell's demons: Does mechanochemical transduction involve a thermal ratchet?" Adv. Biophys. 26, 97-134 (1990)

3 W. Hwang and M. Karplus, "Structural basis for power stroke vs. Brownian ratchet mechanisms of motor proteins," Proc. Natl. Acad. Sci. (USA) 116, 19777-19785 (2019)

${ }^{4}$ W. J. Allen, R. A. Corey, P. Oatley, R. B. Sessions, S. A. Baldwin, S. E. Radford, R. Tuma, and I. Collinson, "Twoway communication between SecY and SecA suggests a Brownian ratchet mechanism for protein translocation," eLife 5, e15598 (2016)

5 R. Ait-Haddou and W. Herzog, "Brownian ratchet models of molecular motors," Cell Biochem. Biophys. 38, 191-213 (2003)

${ }^{6}$ R. D. Astumian and M. Bier, "Fluctuation driven ratchets: Molecular motors," Phys. Rev. Lett. 72, 1766-1769 (1994).

7 H. Wang and G. Oster, "Ratchets, power strokes, and molecular motors," Appl. Phys. A 75, 315-323 (2002)

8 A. Libál, C. Reichhardt, B. Jankó, and C. J. Olson Reichhardt, "Dynamics, rectification, and fractionation for colloids on flashing substrates," Phys. Rev. Lett. 96, 188301
(2006)

${ }^{9}$ S.-H. Lee, K. Ladavac, M. Polin, and D. G. Grier, "Observation of flux reversal in a symmetric optical thermal ratchet," Phys. Rev. Lett. 94, 110601 (2005)

10 C. S. Lee, B. J ankó, I. Derényi, and A. L. Barabási, "Reducing vortex density in superconductors using the "ratchet effect'," Nature (London) 400, 337-340 (1999).

11 R. Wördenweber, P. Dymashevski, and V. R. Misko, "Guidance of vortices and the vortex ratchet effect in high$T_{c}$ superconducting thin films obtained by arrangement of antidots," Phys. Rev. B 69, 184504 (2004).

12 Q. Lu, C. J. Olson Reichhardt, and C. Reichhardt, "Reversible vortex ratchet effects and ordering in superconductors with simple asymmetric potential arrays," Phys. Rev. B 75, 054502 (2007).

${ }^{13}$ W. Gillijns, A. V. Silhanek, V. V. Moshchalkov, C. J. Olson Reichhardt, and C. Reichhardt, "Origin of reversed vortex ratchet motion," Phys. Rev. Lett. 99, 247002 (2007).

14 C. Reichhardt, D. Ray, and C. J. Olson Reichhardt, "Reversible ratchet effects for vortices in conformal pinning arrays," Phys. Rev. B 91, 184502 (2015)

15 N. S. Lin, T. W. Heitmann, K. Yu, B. L. T. Plourde, and V. R. Misko, "Rectification of vortex motion in a circular ratchet channel," Phys. Rev. B 84, 144511 (2011) 
16 C. J. Olson Reichhardt and C. Reichhardt, "Commensurability, jamming, and dynamics for vortices in funnel geometries," Phys. Rev. B 81, 224516 (2010).

17 C. C. de Souza Silva, J. V. de Vondel, M. Morelle, and V. V. Moshchalkov, "Controlled multiple reversals of a ratchet effect," Nature (London) 440, 651-654 (2006).

18 J. E. Villegas, E. M. Gonzalez, M. P. Gonzalez, J. V. Anguita, and J. L. Vicent, "Experimental ratchet effect in superconducting films with periodic arrays of asymmetric potentials," Phys. Rev. B 71, 024519 (2005).

19 C. J. O. Reichhardt and C. Reichhardt, "Rectification and flux reversals for vortices interacting with triangular traps," Physica C 432, 125-132 (2005)

20 J. F. Wambaugh, C. Reichhardt, C. J. Olson, F. Marchesoni, and F. Nori, "Superconducting fluxon pumps and lenses," Phys. Rev. Lett. 83, 5106-5109 (1999)

21 K. Yu, M. B. S. Hesselberth, P. H. Kes, and B. L. T. Plourde, "Vortex dynamics in superconducting channels with periodic constrictions," Phys. Rev. B 81, 184503 (2010)

${ }^{24}$ K. Yu, T. W. Heitmann, C. Song, M. P. DeFeo, B. L. T. Plourde, M. B. S. Hesselberth, and P. H. Kes, "Asymmetric weak-pinning superconducting channels: Vortex ratchets," Phys. Rev. B 76, 220507 (2007).

23 B. L. T. Plourde, "Nanostructured superconductors with asymmetric pinning potentials: Vortex ratchets," IEEE Trans. Appl. Supercond. 19, 3698-3714 (2009).

24 A. Palau, C. Monton, V. Rouco, X. Obradors, and T. Puig, "Guided vortex motion in $\mathrm{YBa}_{2} \mathrm{Cu}_{3} \mathrm{O}_{7}$ thin films with collective ratchet pinning potentials," Phys. Rev. B 85, $012502(2012)$

${ }^{25}$ V. A. Shklovskij and O. V. Dobrovolskiy, "Frequencydependent ratchet effect in superconducting films with a tilted washboard pinning potential," Phys. Rev. B 84, 054515 (2011)

${ }^{20}$ O.V. Dobrovolskiy, E. Begun, V.M. Bevz, R. Sachser, and M. Huth, "Upper frequency limits for vortex guiding and ratchet effects," Phys. Rev. Applied 13, 024012 (2020)

27 Y.-Y. Lyu, J. Jiang, Y.-L. Wang, Z.-L. Xiao, S. Dong, Q.-H. Chen, M. V. Milosevic, H. Wang, R. Divan, J. E. Pearson, P. Wu, F. M. Peeters, and W.-K. Kwok, "Superconducting diode effect via conformal-mapped nanoholes," Nature Commun. 12, 2703 (2021)

28 A. M. Song, "Electron ratchet effect in semiconductor devices and artificial materials with broken centrosymmetry," Appl. Phys. A 75, 229-235 (2002)

29 C. Reichhardt and C. J. Olson Reichhardt, "Active matter ratchets with an external drift," Phys. Rev. E 88, 062310 (2013)

so C. J. Olson Reichhardt and C. Reichhardt, "Ratchet effects in active matter systems," Ann. Rev. Condens. Matter Phys. 8, 51-75 (2017)

${ }^{31}$ D. McDermott, C. J. Olson Reichhardt, and C. Reichhardt, "Collective ratchet effects and reversals for active matter particles on quasi-one-dimensional asymmetric substrates," Soft Matter 12, 8606-8615 (2016).

32 R. Di Leonardo, L. Angelani, D. Dell'Arciprete, G. Ruocco, V. Iebba, S. Schippa, M. P. Conte, F. Mecarini, F. De Angelis, and E. Di Fabrizio, "Bacterial ratchet motors," Proc. Natl. Acad. Sci. (USA) 107, 9541-9545 (2010)

33 C. Reichhardt, D. Ray, and C. J. Olson Reichhardt, "Magnus-induced ratchet effects for skyrmions interacting with asymmetric substrates," New J. Phys. 17, 073034 (2015)
34 W. Chen, L. Liu, Y. Ji, and Y. Zheng, "Skyrmion ratchet effect driven by a biharmonic force," Phys. Rev. B 99, 064431 (2019).

35 W. Chen, L. Liu, and Y. Zheng, "Ultrafast ratchet dynamics of skyrmions by defect engineering in materials with poor conductivity under gigahertz magnetic fields," Phys. Rev. Applied 14, 064014 (2020).

${ }^{36}$ X. Ma, C. J. Olson Reichhardt, and C. Reichhardt, "Reversible vector ratchets for skyrmion systems," Phys. Rev. B 95, 104401 (2017)

37 N. P. Vizarim, C. J. O. Reichhardt, P. A. Venegas, and C. Reichhardt, "Skyrmion pinball and directed motion on obstacle arrays," J. Phys. Commun. 4, 085001 (2020)

38 N.P. Vizarim, C. Reichhardt, P.A. Venegas, and C.J.O. Reichhardt, "Guided skyrmion motion along pinning array interfaces," J. Mag. Mag. Mater. 528, 167710 (2021)

39 B. Göbel and I. Mertig, "Skyrmion ratchet propagation: utilizing the skyrmion Hall effect in AC racetrack storage devices," Sci. Rep. 11, 3020 (2021).

40 V. A. Shklovskij, V. V. Sosedkin, and O. V. Dobrovolskiy, "Vortex ratchet reversal in an asymmetric washboard pinning potential subject to combined dc and ac stimuli," J. Phys.: Condens. Matter 26, 025703 (2014).

${ }^{41}$ L. Dinis, E. M. Gonzalez, J. V. Anguita, J. M. R. Parrondo, and J. L. Vicent, "Lattice effects and current reversal in superconducting ratchets," New J. Phys. 9, 366 (2007)

42 D. Perez de Lara, A. Alija, E. M. Gonzalez, M. Velez, J. I. Martín, and J. L. Vicent, "Vortex ratchet reversal at fractional matching fields in kagomélike array with symmetric pinning centers," Phys. Rev. B 82, 174503 (2010).

43 D. Perez de Lara, M. Erekhinsky, E. M. Gonzalez, Y. J. Rosen, I. K. Schuller, and J. L. Vicent, "Vortex ratchet reversal: Role of interstitial vortices," Phys. Rev. B 83, 174507 (2011)

${ }^{44}$ C. J. Olson Reichhardt and C. Reichhardt, "Commensurability, jamming, and dynamics for vortices in funnel geometries," Phys. Rev. B 81, 224516 (2010).

45 V. Vlasko-Vlasov, T. Benseman, U. Welp, and W. K. Kwok, "Jamming of superconducting vortices in a funnel structure," Supercond. Sci. Technol. 26, 075023 (2013)

46 G. Karapetrov, V. Yefremenko, G. Mihajlović, J. E. Pearson, M. Iavarone, V. Novosad, and S. D. Bader, "Evidence of vortex jamming in Abrikosov vortex flux flow regime," Phys. Rev. B 86, 054524 (2012).

47 N. Nagaosa and Y. Tokura, "Topological properties and dynamics of magnetic skyrmions," Nature Nanotechnol. 8, 899-911 (2013).

${ }^{48}$ F. Jonietz, S. Mühlbauer, C. Pfleiderer, A. Neubauer, W. Münzer, A. Bauer, T. Adams, R. Georgii, P. Böni, R. A. Duine, K. Everschor, M. Garst, and A. Rosch, "Spin transfer torques in MnSi at ultralow current densities," Science 330, 1648-1651 (2010).

${ }^{49}$ T. Schulz, R. Ritz, A. Bauer, M. Halder, M. Wagner, C. Franz, C. Pfleiderer, K. Everschor, M. Garst, and A. Rosch, "Emergent electrodynamics of skyrmions in a chiral magnet," Nature Phys. 8, 301-304 (2012).

50 X. Z. Yu, N. Kanazawa, W. Z. Zhang, T. Nagai, T. Hara, K. Kimoto, Y. Matsui, Y. Onose, and Y. Tokura, "Skyrmion flow near room temperature in an ultralow current density," Nature Commun. 3, 988 (2012).

51 J. Iwasaki, M. Mochizuki, and N. Nagaosa, "Currentinduced skyrmion dynamics in constricted geometries," Nature Nanotechnol. 8, 742-747 (2013). 
52 S.-Z. Lin, C. Reichhardt, C. D. Batista, and A. Saxena, "Driven skyrmions and dynamical transitions in chiral magnets," Phys. Rev. Lett. 110, 207202 (2013).

53 D. Liang, J. P. DeGrave, M. J. Stolt, Y. Tokura, and S. Jin, "Current-driven dynamics of skyrmions stabilized in MnSi nanowires revealed by topological Hall effect," $\mathrm{Na}-$ ture Commun. 6, 8217 (2015)

${ }^{4}$ S. Woo, K. Litzius, B. Krüger, M.-Y. Im, L. Caretta, K. Richter, M. Mann, A. Krone, R. M. Reeve, M. Weigand, P. Agrawal, I. Lemesh, M.-A. Mawass, P. Fischer, M. Kläui, and G. S. D. Beach, "Observation of roomtemperature magnetic skyrmions and their current-driven dynamics in ultrathin metallic ferromagnets," Nature Mater. 15, 501 (2016)

5. Fert, V. Cros, and J. Sampaio, "Skyrmions on the track," Nature Nanotechnol. 8, 152-156 (2013)

56 A. Fert, N. Reyren, and V. Cros, "Magnetic skyrmions: advances in physics and potential applications," Nature Rev. Mater. 2, 17031 (2017)

s7 J. Sampaio, V. Cros, S. Rohart, A. Thiaville, and A. Fert, "Nucleation, stability and current-induced motion of isolated magnetic skyrmions in nanostructures," Nature Nanotechnol. 8, 839-844 (2013).

58 S. Mühlbauer, B. Binz, F. Jonietz, C. Pfleiderer, A. Rosch, A. Neubauer, R. Georgii, and P. Böni, "Skyrmion lattice in a chiral magnet," Science 323, 915-919 (2009).

59 S.-Z. Lin, C. Reichhardt, C. D. Batista, and A. Saxena, "Particle model for skyrmions in metallic chiral magnets: Dynamics, pinning, and creep," Phys. Rev. B 87, 214419 (2013)

ou W. Jiang, X. Zhang, G. Yu, W. Zhang, X. Wang, M. B. Jungfleisch, J. E. Pearson, X. Cheng, O. Heinonen, K. L. Wang, Y. Zhou, A. Hoffmann, and S. G. E. te Velthuis, "Direct observation of the skyrmion Hall effect," Nature Phys. 13, 162-169 (2017).

${ }^{\circ 1}$ K. Litzius, I. Lemesh, B. Krüger, P. Bassirian, L. Caretta, K. Richter, F. Büttner, K. Sato, O. A. Tretiakov, J. Förster, R. M. Reeve, M. Weigand, L. Bykova, H. Stoll, G. Schütz, G. S. D. Beach, and M. Kläui, "Skyrmion Hall effect revealed by direct time-resolved X-ray microscopy,"
Nature Phys. 13, 170-175 (2017).

62 S. Woo, K. M. Song, X. Zhang, Y. Zhou, M. Ezawa, X. Liu, S. Finizio, J. Raabe, N. J. Lee, S. Kim, S.-Y. Park, Y. Kim, J.-Y. Kim, D. Lee, O. Lee, J. W. Choi, B.-C. Min, H. C. Koo, and J. Chang, "Current-driven dynamics and inhibition of the skyrmion Hall effect of ferrimagnetic skyrmions in GdFeCo films," Nature Commun. 9, 959 (2018)

63 R. Juge, S.-G. Je, D. de Souza Chaves, L. D. BudaPrejbeanu, J. Peña Garcia, J. Nath, I. M. Miron, K. G. Rana, L. Aballe, M. Foerster, F. Genuzio, T. O. Menteş, A. Locatelli, F. Maccherozzi, S. S. Dhesi, M. Belmeguenai, Y. Roussigné, S. Auffret, S. Pizzini, G. Gaudin, J. Vogel, and O. Boulle, "Current-driven skyrmion dynamics and drive-dependent skyrmion Hall effect in an ultrathin film," Phys. Rev. Applied 12, 044007 (2019).

64 K. Zeissler, S. Finizio, C. Barton, A. J. Huxtable, J. Massey, J. Raabe, A. V. Sadovnikov, S. A. Nikitov, R. Brearton, T. Hesjedal, G. van der Laan, M. C. Rosamond, E. H. Linfield, G. Burnell, and C. H. Marrows, "Diameter-independent skyrmion Hall angle observed in chiral magnetic multilayers," Nature Commun. 11, 428 (2020)

65 R. Yamaguchi, K. Yamada, and Y. Nakatani, "Control of current-induced skyrmion motion in ratchet-type skyrmion-based racetrack memory with a loop structure," Japan. J. Appl. Phys. 60, 010904 (2020)

66 C. Reichhardt and C. J. O. Reichhardt, "Thermal creep and the skyrmion Hall angle in driven skyrmion crystals," J. Phys.: Condens. Matter 31, 07LT01 (2018)

67 C. Reichhardt, D. Ray, and C. J. O. Reichhardt, "Nonequilibrium phases and segregation for skyrmions on periodic pinning arrays," Phys. Rev. B 98, 134418 (2018).

68 C. Reichhardt and C. J. Olson Reichhardt, "Depinning and nonequilibrium dynamic phases of particle assemblies driven over random and ordered substrates: a review," Rep. Prog. Phys. 80, 026501 (2017).

69 B. Göbel, I. Mertig, and O. A. Tretiakov, "Beyond skyrmions: Review and perspectives of alternative magnetic quasiparticles," Phys. Rep. 895, 1 (2021) 